\title{
Improved Differentiation Ability and Therapeutic Effect of miR-23a-3p Expressing Bone Marrow-Derived Mesenchymal Stem Cells in Mice Model with Acute Lung Injury
}

\author{
Peng Zhang ${ }^{1}$, Linghua Liu ${ }^{2}$, Lei Yao $^{2}$, Xiaoxue Song ${ }^{2}$ \\ ${ }^{I}$ Department of Intensive Care Medicine, Provincial Hospital Affiliated to Shandong First Medical University, Finan, China \\ ${ }^{2}$ Department of Respiratory and Critical Care Medicine, Shaanxi Provincial People's Hospital, Xi'an, China
}

Background and Objectives: Implantation of bone marrow-derived mesenchymal stem cells (BMSCs) has been recognized as an effective therapy for attenuating acute lung injury (ALI). This study aims to discover microRNA (miRNA)-mediated improvement of BMSCs-based therapeutic effects.

Methods and Results: Mice were treated with lipopolysaccharide (LPS) for induction of ALI. BMSCs with lentivirus-mediated expression of miR-23b-3p or fibroblast growth factor 2 (FGF2) were intratracheally injected into the mice with ALI. The expressions of miR-23b-3p, FGF2, Occludin, and surfactant protein C (SPC) in lung tissues were analyzed by immunoblot or quantitative reverse transcription polymerase chain reaction. Histopathological changes in lung tissues were observed via hematoxylin-eosin staining. Lung edema was assessed by the ratio of lung wet weight/body weight (LWW/BW). The levels of interleukin (IL)-1 $\beta$, IL-6, IL-4, and IL-8 in bronchoalveolar lavage fluid (BALF) were assessed by ELISA. LPS injection downregulated the expressions of miR-23b-3p, SPC and Occludin in the lung tissues, increased the LWW/BW ratio and aggravated histopathological abnormalities, while upregulating IL-1 $\beta$, IL-6, IL-4, and IL-8 in the BALF. Upregulated miR-23b-3p counteracted LPS-induced effects, whereas downregulated miR-23b-3p intensified LPS-induced effects. FGF2, which was downregulated by miR-23b-3p upregulation, was a target gene of miR-23b-3p. Overexpressing FGF2 downregulated the expressions of miR-23b-3p, SPC and Occludin, increased the LWW/BW ratio and aggravated histopathological abnormalities, while upregulating IL-1 $\beta$, IL-6, IL-4, and IL-8, and it offset miR-23b-3p upregulation-caused effects on the ALI mice.

Conclusions: Overexpression of miR-23b-3p in BMSCs strengthened BMSC-mediated protection against LPS-induced mouse acute lung injury via targeting FGF2.

Keywords: miR-23b-3p, Acute lung injury, Fibroblast growth factor 2, Lung injury repair, Inflammatory responses

Received: September 3, 2020, Revised: December 16, 2020,

Accepted: January 20, 2021, Published online: February 28, 2021

Correspondence to Linghua Liu

Department of Respiratory and Critical Care Medicine, Shaanxi Provincial People's Hospital, No. 256 Youyi West Road, Beilin District, Xi'an 710068, China

Tel: +86-029-85251331, Fax: +86-029-85236987

E-mail: liulinghua_llh@163.com

(a) This is an open-access article distributed under the terms of the Creative Commons Attribution Non-Commercial License (http://creativecommons.org/ licenses/by-nc/4.0/), which permits unrestricted non-commercial use, distribution, and reproduction in any medium, provided the original work is properly cited.

Copyright (c) 2021 by the Korean Society for Stem Cell Research

\section{Introduction}

Acute lung injury (ALI) is patho-physiologically characterized by alveolar epithelial cell destruction-caused increase in blood-air barrier permeability and non-cardiogenic pulmonary edema (1). Although improvements are seen in the pharmacological and supportive therapies for ALI, the disease still has a high mortality rate of more than $32 \%$ (2). Recent studies have reported that exogenous bone marrow-derived mesenchymal stem cells (MSCs) have a broad biodistribution in different organs including 
ALI lung tissues and can repair damage alveolar epithelium $(3,4)$. Because of their modulating effect on endogenous tissues and cells, MSCs with multipotent differentiation property have been applied in the treatment of multiple clinical disorders such as myocardial infarction, diabetes, and acute renal failure (5). Existing studies have shown that MSCs-based therapy is effective in alleviating human ALI (6). However, the low engraftment and differentiation rates of BMSCs limited their therapeutic effect in clinic (7). Therefore, elucidating the mechanism of promoting the alveolar cell-oriented differentiation may enhance the therapeutic effects of BMSCs implantation on ALI.

MicroRNAs (miRNAs) are a class of single-stranded, endogenous, and non-coding small RNAs that consist of 21-23 nucleotides (8). The function of miRNAs is to degrade mRNAs or inhibit mRNA translation by directly targeting their 3'-untranslated regions (3'-UTRs), which can further affect the proliferation, migration and differentiation of MSCs $(8,9)$, and regulate inflammatory responses (10). More importantly, previous studies have shown that miRNAs like miR-214, miR-16, and miR-23a are dysregulated in LPS (lipopolysaccharide)-induced ALI mouse model (11). MiR-23b-3p is an inflammation-related miRNA and was previously detected to be downregulated in LPS-induced male reproductive tract injury models (12). Moreover, miR-23b-3p upregulation facilitates the hepatic trans-differentiation of MSCs (13). However, the performance of miR-23b-3p in ALI and how miR-23b-3p mediates MSCs-based therapeutic effect on ALI remain obscure.

Fibroblast growth factor 2 (FGF2), which belongs to the family of transcriptional regulator fibroblast growth factors, plays crucial roles in regulating pathological conditions-mediated cell death and inflammatory responses (14). FGF2 can modulate the NF- $\kappa \mathrm{B}$ pathway, which is a typical transcriptional factor that can orchestrate and sustain inflammatory responses by inducing the release of pro-inflammatory mediators and cause tissue damage (15). Cheng et al. (16) discovered that FGF2 regulates inflammation in LPS-exposed alveolar epithelial cells. These lines of evidence suggest that FGF2 inhibition may be a promising strategy to reduce ALI.

In this study, we established LPS-induced ALI models in mice to investigate the relation between miR-23b-3p and FGF2 and strove to propose a miR-23b-3p-related approach to improve the effect of MSCs-based therapy on ALI.

\section{Materials and Methods}

\section{Ethics statement}

All animal experiments were performed in Zhejiang Chinese Medical University in accordance with the guidelines of the China Council on Animal Care and Use. This study was approved by the Committee of Experimental Animals of Zhejiang Chinese Medical University (approval number: ICU201906026). Anesthetics and instant kill were made to minimize the pain and discomfort caused to the animals.

\section{Cell cultures}

Bone marrow-derived mesenchymal stem cells (BMSCs) from $\mathrm{C} 57 \mathrm{BL} / 6$ mice were purchased from Cyagen Biosciences (MUBMX-01001, Santa Clara, CA, USA). The BMSCs were thawed and cultured in OriCell Mouse Mesenchymal Stem Cell Growth Medium (OCMM, MUXMX-90011, Cyagen Biosciences, USA) at $37^{\circ} \mathrm{C}$ in $5 \%$ $\mathrm{CO}_{2}$. After infection with lentivirus carrying miR-27b-3p mimic or FGF2 overexpression plasmid, the BMSCs were passaged in $\mathrm{OCMM}$ at $37^{\circ} \mathrm{C}$ in $5 \% \mathrm{CO}_{2}$. Briefly, when the BMSCs grew to a monolayer of $80 \sim 90 \%$ confluence, the media were aspirated. The BMSCs were rinsed with phosphate saline buffer (PBS, P5493, Sigma-Aldrich, St. Louis, MO, USA) for two or three times and then detached by 0.25\% Trypsin-0.04\% EDTA (T4049 Sigma-Aldrich, USA). The detached BMSCs were resuspended by OCMM and transferred to new flasks. The 6th or 7th passage of BMSCs was used for the following experiments except lentivirus infection.

293 T cells were purchased from Procell (CL-0005, Wuhan, China) and cultured in Dulbecco's Modified Eagle Media (A4192101, ThermoFisher, Waltham, MA, USA) supplemented with $10 \%$ fetal bovine serum (FBS, F8687, Sigma-Aldrich, USA), $1 \%$ penicillin/streptomycin (V900929, Sigma-Aldrich, USA) and 1\% Glutamine (G7513, Sigma-Aldrich, USA).

\section{Lentiviral vector construction, packaging, and infection}

Primers for miR-23b-3p overexpression (forward: 5'-CC TCGAGGTACCGTCGTATCCAGTGCAA-3', reverse: 5'GGGTACCCCGTATCCAGTGCGTGTCGT-3'), miR-23b3p underexpression (forward: 5'-GGGACCGGGGTCCCT AATGGCAGCATAG-3', reverse: 5'-GGAATTCCATAGGT CACGCACAGCACCT-3'), and FGF2 overexpression (forward: 5'-CCTCGAGGGCGACCCACACGTCAAACTA-3', reverse: 5'-GGGTACCCTCCCTTGATAGACACAACTCC TC-3') were used to produce the corresponding coding sequences. The coding sequence of miR-23b-3p overex- 
pression or FGF2 overexpression was cloned into pLVXIRES-ZsGreenl (Hedgehogbio, Shanghai, China) which had been digested by Xhol and Kpnl endonucleases (FD0694 and ER0521, ThermoFisher, USA). The coding sequence of miR-23b-3p underexpression was cloned into GV280 (Genechem, Shanghai, China) which had been digested by AgeI and EcoRI endonucleases (ER1461 and FD0274, ThermoFisher, USA). Empty lentiviral vectors were used as negative control. $293 \mathrm{~T}$ cells were seeded at $70 \%$ confluence in 12-well plates. Then, the $293 \mathrm{~T}$ cells were cotransfected either with the recombinant pLVX-IRESZsGreen1 vector, plp1, plp2 and plp/vsvg or with the recombinant GV280 vector, pGC-LV, Helper1.0 and Helper2.0 using Lipofectamine 3000 Transfection Reagent (L3000015, ThermoFisher, USA). Forty-eight hours after transfection, the culture media were collected. Viral particles were obtained via centrifugal ultrafiltration. Then, the viral particles were diluted and used to infect BMSCs.

\section{Preparation of animals and establishment of ALI models}

Fifty-four 6- to 8-week-old male C57BL/6 mice (20 25g) were purchased from ALF Biotechnology (Jiangsu, China). All the mice were maintained at $20 \sim 24^{\circ} \mathrm{C}$ and $50 \%$ humidity on a $12 \mathrm{~h} / 12 \mathrm{~h}$ light/dark cycle, and were given free access to food and water. Prior to experimentation, the mice were acclimated for 3 days.

All the mice were anesthetized via intraperitoneal injection of 2\% pentobarbital sodium (P-010, Sigma-Aldrich, USA). For establishment of ALI models, the mice were intratracheally injected with a single dose of $100 \mu \mathrm{g}$ lipopolysaccharide (LPS, L2630, Sigma-Aldrich, USA) which was previously dissolved in $50 \mu 1$ normal saline (NS). The mice were allowed to recover until fully awake. The lentivirus-infected BMSCs $\left(5 \times 10^{4}\right.$ cells $)$ were resuspended in $30 \mu 1$ of PBS for implantation therapy.

\section{Animal grouping}

All the mice were randomly allocated into nine groups $(n=6)$ and received different treatments as follows. Control group: mice received no further treatment; LPS group: 4 hours after modeling, ALI mice underwent intratracheal installation of $30 \mu 1$ of PBS; LPS + NC group: ALI mice were intratracheally injected with BMSCs infected with lentivirus carrying negative control $\left(5 \times 10^{4}\right.$ cells in $30 \mu 1$ of PBS; MOI $=10)$; $L P S+M$ group: ALI mice were intratracheally injected with BMSCs infected with lentivirus carrying miR-23b-3p mimic $\left(5 \times 10^{4}\right.$ cells in $30 \mu 1$ of PBS); LPS + I group: ALI mice were intratracheally injected with BMSCs infected with lentivirus carrying miR-23b-3p inhibitor $\left(5 \times 10^{4}\right.$ cells in $30 \mu 1$ of PBS); NC+FGF2-NC group: ALI mice were intratracheally injected with BMSCs infected with lentivirus carrying mimic control and FGF2-negative control; $\mathrm{M}+$ FGF2-NC group: ALI mice were intratracheally injected with BMSCs infected with lentivirus carrying miR-23b-3p mimic and FGF2-negative control; NC+FGF2 group: ALI mice were intratracheally injected with BMSCs infected with lentivirus carrying mimic control and FGF2 overexpression plasmid; $M+F G F 2$ group: ALI mice were intratracheally injected with BMSCs infected with lentivirus carrying miR-23b-3p mimic and FGF2 overexpression plasmid. Lastly, all the mice were sacrificed via spinal dislocation, and their lung tissues were collected and stored at $-80^{\circ} \mathrm{C}$ until use in the following experiments.

\section{Dual-luciferase reporter assay}

The putative binding sites of miR-23b-3p on FGF2 transcripts were provided by Targetscan7.2 (http://www. targetscan.org/vert_72/), an online prediction tool. A reporter plasmid containing the 3' UTR of FGF2-Wild-Type (5'-TGGTGTTATTTAGTTAATGTGAT-3') or FGF2-MutantType (5'-TGGTGTTATTTAGTTGGCTCACG-3') was constructed using pmirGLO vectors (E1330, Promega, Madison, WI, USA). BMSCs were seeded in 12-well plates (140656, ThermoFisher, USA) at $37^{\circ} \mathrm{C}$ with $5 \% \mathrm{CO}_{2}$. When reaching $70 \%$ confluence, the BMSCs were co-transfected with the reporter plasmid and either miR-23b-3p mimic (3'CCAUUAGGGACCGUUACACUA-5', miR10000891-1-5, RIBOBIO, China) or mimic control (3'-CACACACACA CAUGUGUGUGU-5', miR10000125-1-5, RIBOBIO, China) using Lipofectamine 3000 Transfection Reagent. After transfection for 48 hours, the luciferase activity of the reporter plasmid was detected by a dual-Luciferase Reporter Assay System (E1980, Promega, USA).

\section{Evaluation of lung edema}

The ratio of lung wet weight to body weight (LWW/BW) was calculated and recorded to indicate the severity of lung edema. Briefly, the collected mouse lung tissues were trimmed for removal of extrapulmonary tissues, and then lung wet weight and body weight were measured. The results were presented as milligram per gram.

\section{Quantitative reverse transcription polymerase chain reaction (qRT-PCR)}

Total RNA and miRNA were extracted from lung tissues of ALI mice using Trizol reagent (15596026, ThermoFisher, USA) and PureLink miRNA Isolation Kits (K157001, ThermoFisher, USA), respectively. The total 
RNA and total miRNA were purified by successive treatments with chloroform (151858, Sigma-Aldrich, USA), isopropanol (I9516, Sigma-Aldrich, USA), and 75\% ethanol (E7023, Sigma-Aldrich, USA). The purified total mRNA and miRNA underwent reverse transcription reaction with SuperScript IV reverse transcriptase (18090010, ThermoFisher, USA) for cDNA synthesis. A Real-Time PCR detection System (CFX Connect, Bio-Rad, Philadelphia, PA, USA) and PowerUp SYBR Green Master Mix (A25742, ThermoFisher, USA) were used for product amplification. The primers used for the amplification were listed in Table 1 . The reaction conditions were: $95^{\circ} \mathrm{C}$ pre-denaturation for $10 \mathrm{~min}, 95^{\circ} \mathrm{C}$ denaturation for $15 \mathrm{~s}$, and 40 circles of $95^{\circ} \mathrm{C}$ annealing for $15 \mathrm{~s}$ and $60^{\circ} \mathrm{C}$ elongation for $60 \mathrm{~s}$. The relative expression levels were calculated by the $2^{-\Delta \Delta \mathrm{Ct}}$ method (17).

\section{Immunoblot}

Total protein lysates were prepared from lung tissues of ALI mice using RIPA Buffer (89900, ThermoFisher, USA) and quantitated using a BCA protein assay kit (A53227, ThermoFisher, USA). Forty microliter of protein and $5 \mu 1$ of marker (PR1910, Solarbio, China) were separately loaded on 12\% SDS-PAGE gel (P0053A, Beyotime, Shanghai, China) and subjected to electrophoresis. Afterwards, the protein was transferred onto PVDF membranes (P2438, Sigma-Aldrich, USA), and the membranes were blocked by Tris Buffered Saline and 1\% Tween 20 (TBST, TA125-TT, ThermoFisher, USA) containing 5\% skimmed milk. Then, the membranes were incubated at $4{ }^{\circ} \mathrm{C}$ overnight with primary antibodies against Occludin (SAB35 00301, $57 \mathrm{kDa}, 1:$ 1,000, Sigma-Aldrich, USA), SPC (ab2 11326, $21 \mathrm{kDa}, 1$ : 1,000, Abcam, Cambridge, MA, USA), FGF2 (SAB2108135, $31 \mathrm{kDa}, 1:$ 1,000, Sigma-Aldrich, USA), and GAPDH (ab181602, $36 \mathrm{kDa}, 1:$ 10,000, Abcam, USA). Then, the membranes were incubated with Goat Anti-Rabbit IgG (A32733, $1:$ 20,000, ThermoFisher, USA) at room temperature for 1 hour. The immunoreactive bands were detected on an imaging system (iBright CL1500, A44240, ThermoFisher, USA) using an enhanced chemiluminescence reagent kit (WP20005, ThermoFisher, USA). The density of the bands was quantified by ImageJ software (version 1.52s, National Institutes of Health, Bethesda, MD, USA).

\section{Histopathology}

Lung tissues of ALI mice were fixed in $10 \%$ paraformaldehyde (16005, Sigma-Aldrich, USA) for $24 \mathrm{~h}$, dehydrated by gradient alcohol, transparentized by xylene (95682, Sigma-Aldrich, USA), embedded in paraffin (1496904, SigmaAldrich, USA) and cut into 5- $\mu \mathrm{m}$ thick sections. Then, after being dewaxed by xylene and rehydrated by gradient alcohol, the sections were stained with Hematoxylin (H3136, Sigma-Aldrich, USA) for $12 \mathrm{~min}$ and eosin for 5 min. After staining, the sections were sealed with neutral balsam (N861409, Macklin, Shanghai, China) and dried at $37^{\circ} \mathrm{C}$ for 4 hours. The histopathological changes of the lung tissues were observed by an optical microscope (13132071, fisherscientific, Massachusetts, USA) under 100× magnification.

\section{Enzyme-linked immunosorbent assay (ELISA)}

A tracheal cannula was passed through the nose into the lungs of ALI mice. Then, the tracheal cannula was withdrawn and flushed by pre-cooled PBS thrice. The flushing fluid was centrifuged at $1,000 \times \mathrm{g}$ at $4{ }^{\circ} \mathrm{C}$ to obtain bronchoalveolar lavage fluid (BALF). The levels of interleukin (IL)- $1 \beta$, IL-6, IL-4 and IL-8 in the BALF were measured by their corresponding ELISA kits (KGEMC001b, KGE MC004, KGEMC003, KGEMC104, keygentec, Jiangsu, China) according to the instruction of the manufacturer. Briefly, the BALF samples were diluted by normal saline, added into ELISA plates (100 $\mu \mathrm{l} /$ well) and incubated at $36^{\circ} \mathrm{C}$ for $90 \mathrm{~min}$. After being washed by PBS, the ELISA plates were added with biotinylated antibodies (100 $\mu \mathrm{l} /$ well) and incubated at $36^{\circ} \mathrm{C}$ for $60 \mathrm{~min}$. After being washed by PBS again, the ELISA plates were added with enzyme conjugation working solution (100 $\mu \mathrm{l} /$ well) and incubated at $36^{\circ} \mathrm{C}$ for $60 \mathrm{~min}$ in the darkness. Lastly, Tetramethylbenzidine was added into the ELISA plates for color

Table 1. Primers used in quantitative reverse transcription polymerase chain reaction for the target genes

\begin{tabular}{|c|c|c|c|}
\hline Gene & Species & Forward & Reverse \\
\hline miR-23b-3p & Mouse & 5'-ATTGCCAGGGATTACCGTCG-3' & 5'-GTGTCGTGGAGTCGGCAATT-3' \\
\hline FGF2 & Mouse & 5'-GCGACCCACACGTCAAACTA-3' & 5'-TCССТTGATAGACACAACTCCТC-3' \\
\hline SPC & Mouse & 5'-ATGGACATGAGTAGCAAAGAGGT-3' & 5'-CACGATGAGAAGGCGTTTGAG-3' \\
\hline Occludin & Mouse & 5'-TTGAAAGTCCACCTCCTTACAGA-3' & 5'-CCGGATAAAAAGAGTACGCTGG-3' \\
\hline U6 & Mouse & 5'-CTCGCTTCGGCAGCACATATACT-3' & 5'-ACGCTTCACGAATTTGCGTGTC-3' \\
\hline GAPDH & Mouse & 5'-TGGTGAAGCAGGCATCTGAG-3' & 5'-TGAAGTCGCAGGAGACAACC-3' \\
\hline
\end{tabular}


development. The reaction was terminated using $2 \mathrm{~mol} / \mathrm{l}$ $\mathrm{H}_{2} \mathrm{SO}_{4}$, and optical density was read under a microplate reader (ELx808, BioTek, Vermont, USA) at a wavelength of $450 \mathrm{~nm}$.

\section{Statistical analysis}

All data were obtained from at least three independent experiments and are expressed as the mean \pm standard deviation. Statistical analyses were performed using SPSS 22.0 software (IBM, Armonk, NY, USA). Comparisons among multiple groups and between two groups were performed using one-way analysis of variance and student t-test respectively, and both were followed by Bonferroni's post-hoc test. $\mathrm{p}<0.05$ was considered statistically significant.

\section{Results}

\section{MiR-23b-3p was low-expressed in the lung tissues of ALI mice}

To investigate the impact of implantation of BMSCs with manipulated miR-23b-3p expression on ALI, LPS-induced mice models of ALI were injected with BMSCs infected with lentivirus carrying miR-23b-3p mimic or inhibitor. qRT-PCR analysis showed that compared to the Control group, miR-23b-3p expression was decreased after LPS injection $(p<0.001)$, while implantation of BMSCs overexpressing miR-23b-3p increased miR-23b-3p expression in ALI lung tissues, compared to the ALI mice without implantation $(\mathrm{p}<0.001$, Fig. 1A). However, implantation of BMSCs under-expressing miR-23b-3p exerted no obvious effects on miR-23b-3p expression in ALI lung tissues.

\section{MiR-23b-3p-overexpressing BMSCs promoted the differentiation of BMSCs, increased lung epithelium permeability, and improved lung edema}

Subsequently, the expressions of SPC (a marker of TypeII pneumocytes), Oct4, Nanog (stemness marker) and Occludin (a marker of lung permeability) in ALI lung tissues were analyzed, and the LWW/BW ratio was calculated. Occludin expression is associated with the status of the tight junctions of the lung epithelium (18). qRT-PCR and immunoblot analyses and lung edema evaluation revealed that LPS injection downregulated the mRNA and protein expression levels of SPC, Oct4, Nanog and Occludin and increased the LWW/BW ratio, compared to the Control group $(p<0.001)$, while compared to the ALI mice without implantation, implantation of BMSCs overexpressing miR-23b-3p increased the expression levels of
SPC, Oct4, Nanog and Occludin and decreased the LWW/BW ratio, and implantation of BMSCs under-expressing miR-23b-3p decreased the expression levels of SPC, Oct4, Nanog and Occludin and increased LWW/BW in ALI lung tissues $(p<0.05, p<0.01, p<0.001$, Fig. $1 B \sim$ I).

\section{MiR-23b-3p-overexpressing BMSCs inhibited inflammatory factor release in ALI lung tissues}

Later, changes in histopathology and inflammatory factor levels were detected in ALI lung tissues by Hematoxylin-eosin staining and ELISA. As presented in Fig. 1J $\mathrm{P}$, LPS injection caused inflammatory infiltration, structural damage, and increased levels of IL-1 $\beta$, IL-6, IL-4, IL-8, TNF- $\alpha$ and IL-10, compared to the control group $(\mathrm{p}<0.001)$. Implantation of BMSCs overexpressing miR$23 \mathrm{~b}-3 \mathrm{p}$ alleviated inflammatory infiltration and structural damage and lowered the levels of IL-1 $\beta$, IL-6, IL-4, IL-8 and TNF- $\alpha$, while increased the level of IL-10, compared to the ALI mice without implantation $(\mathrm{p}<0.001)$, while implantation of BMSCs under-expressing miR-23b-3p aggravated inflammatory infiltration and structural damage and elevated the levels of these inflammatory factors (Fig. $1 \mathrm{~J} \sim \mathrm{P})$.

\section{MiR-23b-3p directly targeted FGF2}

Bioinformatics analysis conducted on TargetScan V7.2 predicted that there existed highly conserved putative binding sites of miR-23b-3p on the 3'-UTR of FGF2-wild type (Fig. 2A). To validate the binding relation between miR-23b-3p and FGF2, we performed dual-luciferase reporter assay. The results showed that BMSCs co-transfected with the 3'UTR of FGF2-wild-type and miR-23b-3p mimic displayed a decreased luciferase activity, compared to those co-transfected with the 3'UTR of FGF2-wild-type and NC $(p<0.001$, Fig. 2B). However, transfection of miR-23b-3p mimic into the BMSCs loaded with FGF2mutant type did not produce obvious changes in luciferase activity (Fig. 2B). These data suggested that miR-23b-3p could interact with FGF2 by directly binding to the 3'-UTR of FGF2.

\section{FGF2 expression was inhibited by miR-23b-3p overexpressing BMSCs, and it inhibited the differentiation of BMSCs into Typell pneumocytes}

The role of the interaction between FGF2 and miR23b-3p in ALI was examined via implantation of BMSCs infected with lentivirus carrying both miR-23b-3p mimic and FGF2 overexpression plasmid into ALI mice. As revealed by qRT-PCR and immunoblot analyses, miR-23b- 


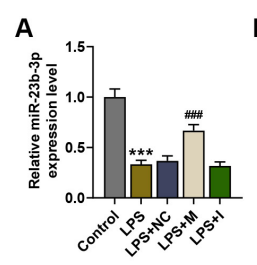

B

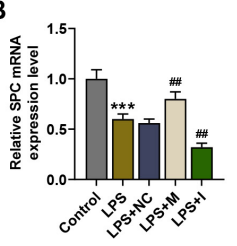

D

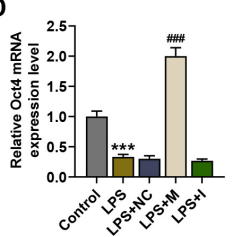

C

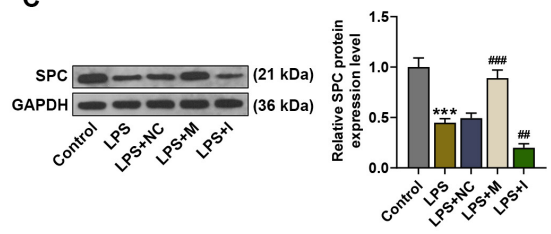

E

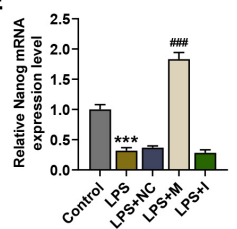

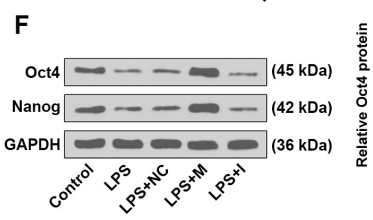
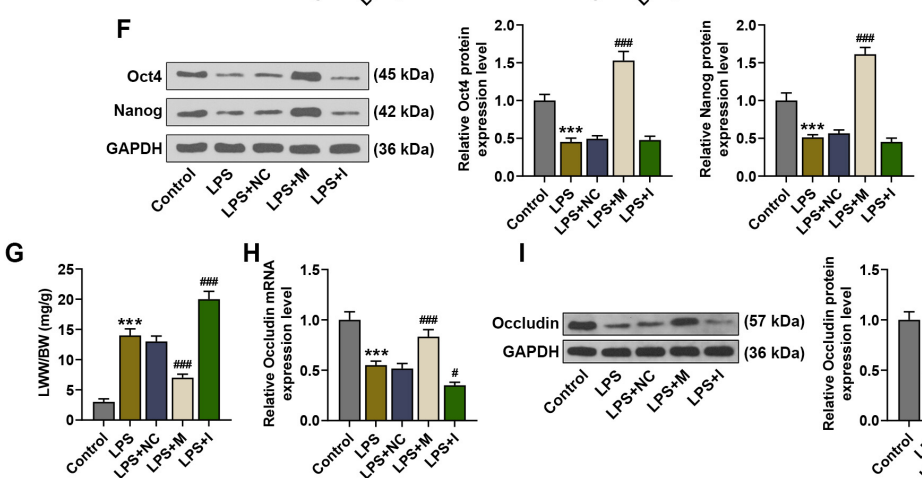

I

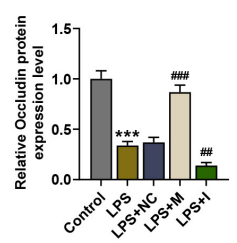

J

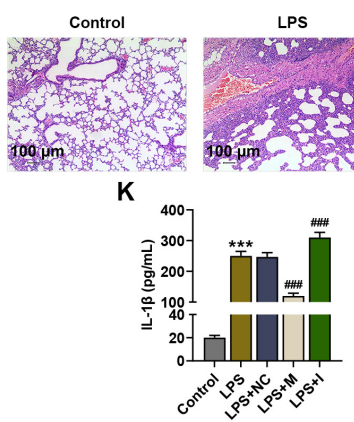

N

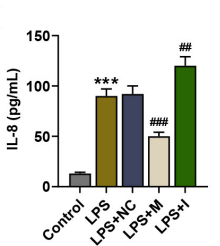

L
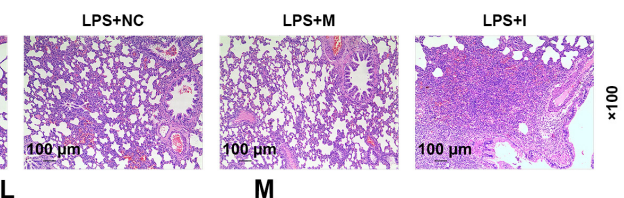

M
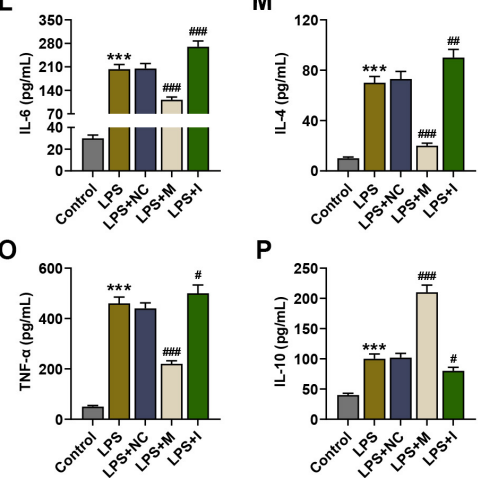

Fig. 1. MiR-23b-3p-overexpressing BMSCs promoted the differentiation of BMSCs into Typell pneumocytes and alleviated injuries in ALI lung tissues. (A, B, D, E, H) The expressions of miR-23b-3p, SPC, Oct4, Nanog and Occludin in the lung tissues of LPS-induced ALI mice were analyzed by qRT-PCR, after injection of BMSCs with no altered gene expression or BMSCs with miR-27a-3p overexpression or underexpression. (C, F, I) The expressions of SPC, Oct4, Nanog and Occludin in the lung tissues of LPS-induced ALI mice were analyzed by immunoblot, with GAPDH serving as a reference gene, after injection of BMSCs with no altered gene expression or BMSCs with miR-27a-3p overexpression or underexpression. (G) The ratio of LWW to BW of LPS-induced ALI mice was calculated, after injection of BMSCs with no altered gene expression or BMSCs with miR-27a-3p overexpression or underexpression. (J) Histopathological changes in the lung tissues of LPS-induced ALI mice were observed via hematoxylin-eosin staining, after injection of BMSCs with no altered gene expression or BMSCs with miR-27a-3p overexpression or underexpression (scale: $100 \mu \mathrm{m}$; magnification: $\times 100$ ). $(\mathrm{K} \sim \mathrm{P})$ The levels of IL-1 $\beta$, IL-6, IL-4, IL-8, TNF- $\alpha$ and IL-10 in the bronchoalveolar lavage fluid of LPS-induced ALI mice were assessed by ELISA, after injection of BMSCs with no altered gene expression or BMSCs with miR-27a-3p overexpression or underexpression. ${ }^{\#} \mathrm{p}<0.05 ;{ }^{\# \#} \mathrm{p}<0.01$; ${ }^{* * *} \mathrm{p}$ or $\#$ \# $<0.001$; * vs. Control ; \# vs. LPS + NC (LPS: lipopolysaccharide, SPC: surfactant protein C, NC: negative control, M: miR-23b-3p mimic, I: miR-23b-3p inhibitor, qRT-PCR: Quantitative reverse transcription polymerase chain reaction, ELISA: Enzyme-linked immunosorbent assay, ALI: acute lung injury, LWW/BW: lung wet weight/body weight, BMSCs: bone marrow-derived mesenchymal stem cells). 
3p-overexpressing BMSCs inhibited the mRNA and protein levels of FGF2, while implantation of BMSCs overexpressing FGF2 promoted these levels, compared to the $\mathrm{NC}+\mathrm{FGF} 2-\mathrm{NC}$ group $(\mathrm{p}<0.001$, Fig. $3 \mathrm{~A}$ and $3 \mathrm{~B})$. Moreover, FGF2 mRNA and protein expressions under the im-

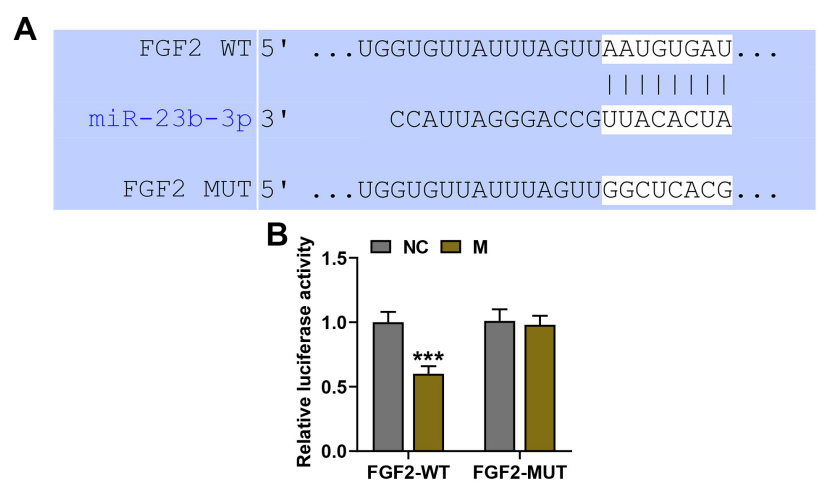

Fig. 2. MiR-23b-3p directly targeted FGF2. (A) The putative binding sites of miR-23b-3p on FGF2 were predicted by TargetScan V7.2. (B) The interaction between miR-23b-3p and FGF2 was validated by dual-luciferase reporter assay. ${ }^{* * *} p<0.001 ;{ }^{*}$ vs. NC (NC: negative control, M: miR-23b-3p mimic, WT: wild type, MUT: mutant type, FGF2: fibroblast growth factor 2). plantation of BMSCs co-overexpressing miR-23b-3p and FGF2 were lower than those under the implantation of BMSCs overexpressing FGF2 alone $(p<0.001)$, but higher than those under the implantation of BMSCs overexpressing miR-23b-3p alone $(p<0.01, p<0.001$, Fig. $3 \mathrm{~A}$ and $3 \mathrm{~B})$. Furthermore, SPC mRNA and protein expressions were increased after implantation of BMSCs overexpressing miR-23b-3p, while they were decreased after implantation of BMSCs overexpressing FGF2, compared to the NC+FGF2-NC group $(p<0.01, p<0.001$, Fig. 3C and 3D). Moreover, SPC mRNA and protein expressions under the implantation of BMSCs co-overexpressing miR-23b-3p and FGF2 were higher than those under the implantation of BMSCs overexpressing FGF2 alone $(\mathrm{p}<0.001)$, but lower than those under the implantation of BMSCs overexpressing miR-23b-3p alone $(\mathrm{p}<$ $0.05, \mathrm{p}<0.01$, Fig. 3C and 3D).

\section{FGF2 reversed the effect of the implantation of BMSCs overexpressing miR-23b-3p on ALI lung tissues}

Then, the relation between miR-23b-3p-mediated changes in FGF2 expression and ALI progression was further explored. As compared to the NC+FGF2-NC group,
A

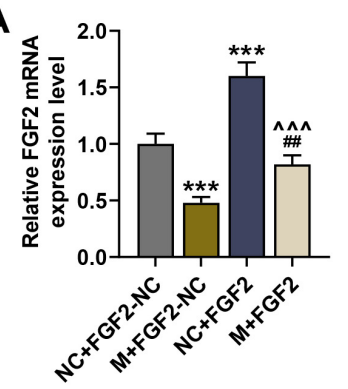

C

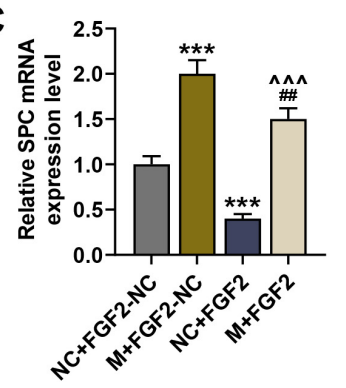

B
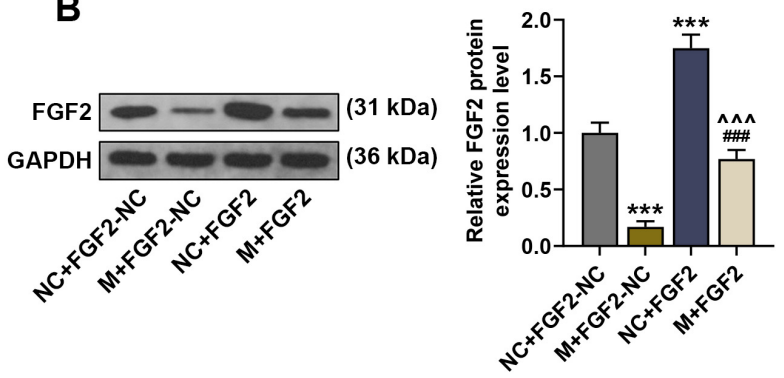

D

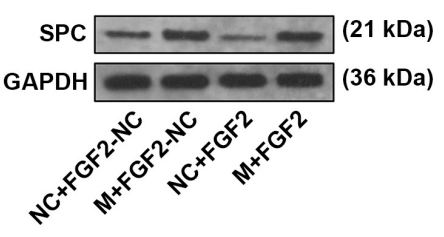

Fig. 3. FGF2 expression was inhibited by miR-23b-3p overexpressing BMSCs and it inhibited the differentiation of BMSCs into Typell pneumocytes. (A, C) The expressions of FGF2 and SPC in the lung tissues of LPS-induced ALI mice were analyzed by qRT-PCR, after injection of BMSCs with no altered gene expression, BMSCs with overexpression of miR-27a-3p or FGF2 alone, or BMSCs with co-overexpression of miR-27a-3p and FGF2. (B, D) The expressions of FGF2 and SPC in the lung tissues of LPS-induced ALI mice were analyzed by immunoblot, with GAPDH serving as a reference gene, after injection of BMSCs with no altered gene expression, BMSCs with overexpression of miR-27a-3p or FGF2 alone, or BMSCs with co-overexpression of miR-27a-3p and FGF2. ${ }^{*} \mathrm{p}<0.05 ;{ }^{* *} \mathrm{p}$ or ${ }^{\# \#} \mathrm{p}<0.01 ;{ }^{* * *} \mathrm{p}$

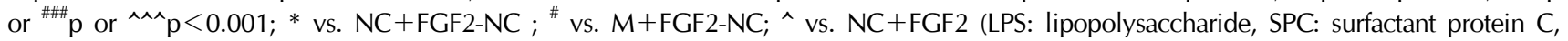
FGF2: fibroblast growth factor 2, NC: negative control, M: miR-23b-3p mimic, qRT-PCR: Quantitative reverse transcription polymerase chain reaction, BMSCs: bone marrow-derived mesenchymal stem cells). 
implantation of BMSCs overexpressing FGF2 alone increased the LWW/BW ratio and downregulated the mRNA and protein levels of Occludin (Fig. 4A C), and it reversed the effect of miR-23b-3p upregulation on lowering the LWW/BW ratio and promoting Occludin expression. Meanwhile, implantation of BMSCs co-over- expressing miR-23b-3p and FGF2 reversed the inhibitory effect of miR-23b-3p upregulation on the LWW/BW ratio and the inhibitory effect of FGF2 upregulation on Occludin expression (Fig. $4 \mathrm{~A} \sim \mathrm{C}$ ). Furthermore, implantation of BMSCs overexpressing FGF2 alone caused inflammatory infiltration and structural damage and ele-
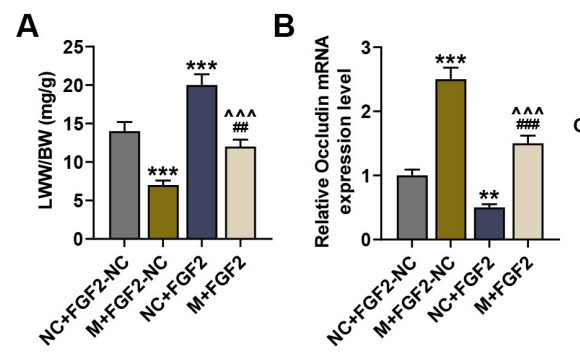

C

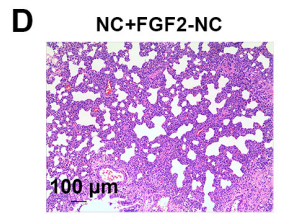

M+FGF2-NC
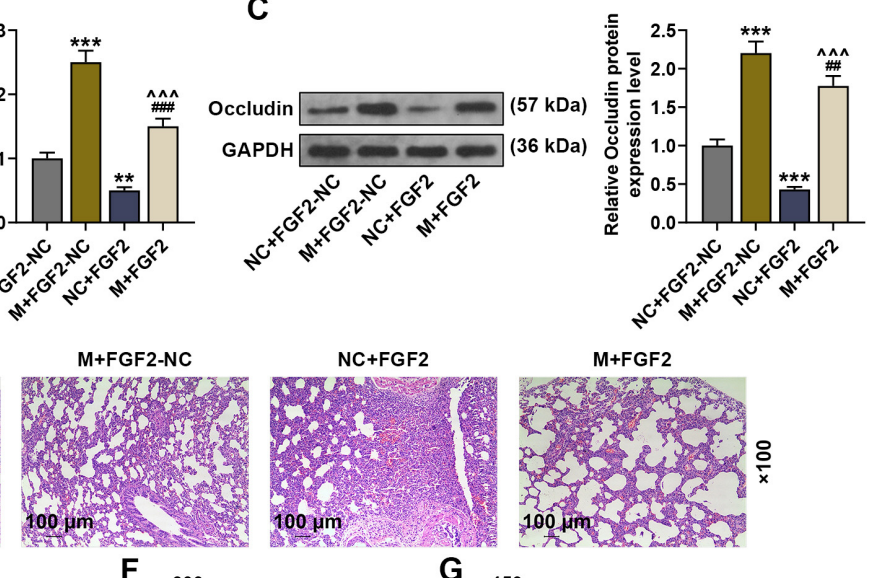

M+FGF2
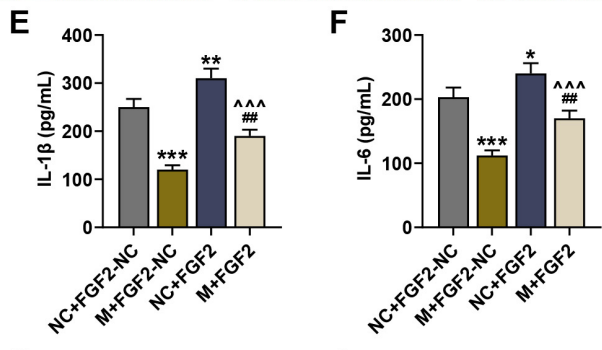

G

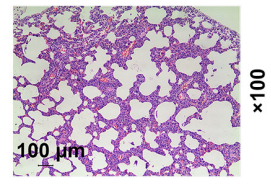

H

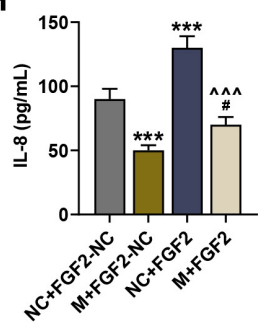

I

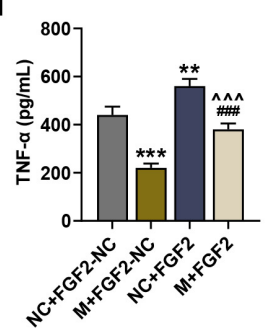

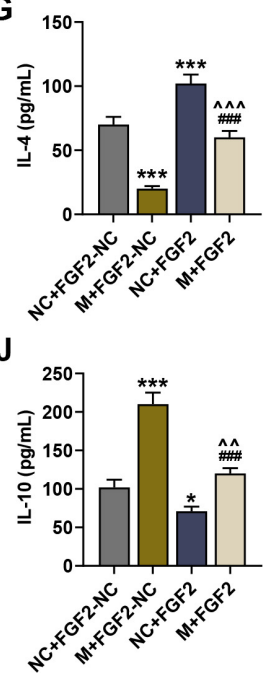

Fig. 4. FGF2 reversed the effect of the implantation of BMSCs overexpressing miR-23b-3p on ALI lung tissues. (A) The ratio of LWW to BW of LPS-induced ALI mice was calculated, after injection of BMSCs with no altered gene expression, BMSCs with overexpression of miR-27a-3p or FGF2 alone, or BMSCs with co-overexpression of miR-27a-3p and FGF2. (B) The expression of Occludin in the lung tissues was analyzed by qRT-PCR, after injection of BMSCs with no altered gene expression, BMSCs with overexpression of miR-27a-3p or FGF2 alone, or BMSCs with co-overexpression of miR-27a-3p and FGF2. (C) The expression of Occludin in the lung tissues of LPS-induced ALI mice was analyzed by immunoblot, with GAPDH serving as a reference gene, after injection of BMSCs with no altered gene expression, BMSCs with overexpression of miR-27a-3p or FGF2 alone, or BMSCs with co-overexpression of miR-27a-3p and FGF2. (D) Histopathological changes in the lung tissues of LPS-induced ALI mice were observed via hematoxylin-eosin staining, after injection of BMSCs with no altered gene expression, BMSCs with overexpression of miR-27a-3p or FGF2 alone, or BMSCs with co-overexpression of miR-27a-3p and FGF2 (scale: $100 \mu \mathrm{m}$; magnification: $\times 100$ ). (E J) The levels of IL-1 $\beta$, IL-6, IL-4, IL-8, TNF- $\alpha$ and IL-10 in the bronchoalveolar lavage fluid of LPS-induced ALI mice were assessed by ELISA, after injection of BMSCs with no altered gene expression, BMSCs with overexpression of miR-27a-3p or FGF2 alone, or BMSCs with co-overexpression of miR-27a-3p and FGF2. ${ }^{*} p$ or ${ }^{\#} p<0.05 ;{ }^{* *} p$ or ${ }^{\# \#} p$ or ${ }^{\wedge} p<0.01 ;{ }^{* * *} p$

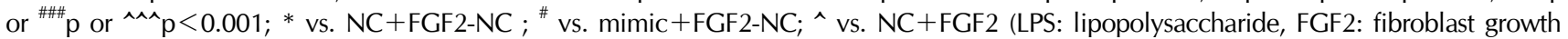
factor 2, NC: negative control, M: miR-23b-3p mimic, qRT-PCR: Quantitative reverse transcription polymerase chain reaction, ELISA: Enzyme-linked immunosorbent assay, ALI: acute lung injury, LWW/BW: lung wet weight/body weight, BMSCs: bone marrow-derived mesenchymal stem cells). 
vated the levels of IL- $1 \beta$, IL-6, IL-4, IL-8 and TNF- $\alpha$, while decreased IL-10 level, compared to the NC+FGF2NC group $(p<0.05, p<0.01, p<0.001)$, and it reversed the inhibitory effect of miR-23b-3p upregulation on histopathological abnormalities and inflammatory factor release (Fig. 4D J). Meanwhile, implantation of BMSCs overexpressing miR-23b-3p reversed the promotive effect of FGF2 upregulation on histopathological abnormalities and inflammatory factor release (Fig. $4 \mathrm{D} \sim \mathrm{J}$ ).

\section{Discussion}

BMSCs-based therapy is currently deemed as a feasible strategy for the treatment of ALI, during which BMSCs migrate to injured lungs to further differentiate into alveolar epithelial cells and facilitate the retention of the cells in the lung $(3,4)$. Recent studies have demonstrated that regulating gene expression in MSCs that are used in the implantation therapy for ALI can inhibit cell apoptosis, oxidative stress and inflammatory response and promote the migration of MSCs and their differentiation into TypeII pneumocytes in injured lung tissues, suggesting that MSCs may enhance BMSCs-mediated protection of lung tissues $(19,20)$. However, the low engraftment and differentiation rates limit the therapeutic potential of MSCs-based therapy (21). Therefore, finding a method to promote the migration and differentiation of MSCs is in urgent need. In this study, we revealed that miR-23b-3p mediated inhibition of FGF2 enhanced alveolar cell-oriented differentiation and inhibited the release of inflammatory factors, thus contributing to lung tissue repair in ALI.

MiRNAs are present in the circulatory system and changes in miRNA expression may herald the development of a variety of diseases including ALI (22, 23). Cardinal-Fernández et al. (24) have proposed that miRNAs are suitable biomarkers and therapeutic targets for ALI. As reported by Suo et al. (25), miR-1246 expression is significantly increased in ALI mice, whereas knockdown of miR-1246 inhibited cell apoptosis and inflammation in an in vitro ALI model. In contrast to our findings, Zhang et al. (26) discovered that miR-23b was downregulated in LPS-induced injury models and overexpressing miR-23b alleviated LPS-induced inflammatory responses. In addition, miR-23a-3p downregulation was previously found to be associated with LPS-induced rat testis (12), while miR-23a-3p upregulation was detected in acute laminitis horses (27). Our study demonstrated that LPS-induced ALI markedly downregulated miR-23a-3p expression, and similar to Dong's findings (28), it also increased LWW/BW, promoted inflammatory infiltration and enhanced inflammatory factor release in vivo.

Meanwhile, Wang's study demonstrated that the alleviation of LPS-induced ALI caused by lung-resident MSCs displayed an increased expression of SPC and a reduced number of inflammatory cytokines (29). SPC, an essential component of pulmonary surfactant, is primarily expressed in TypeII pneumocytes and has an anti-inflammation property, and SPC deficiency prompts the fast development of lung injury (30). Occludin plays a crucial role in assembling or maintaining epithelial tight junctions and regulating the barrier function of tight junctions (31). Deterioration of Occluding resulted in impaired function of alveolar epithelial barrier, which predisposes people to ALI (32). We also detected downregulated expressions of SPC and Occludin after LPS injection.

As emphasized above, lung tissue repair requires the retention of exogenous BMSCs in the damaged sites on tissues (33). We investigated BMSCs implantation-delivered therapeutic effect of miR-23a-3p on tissue damage and inflammatory responses in ALI. Our study discovered that injection of miR-23a-3p-overexpressing BMSCs into ALI mice not only restored miR-23a-3p expression, but also decreased the LWW/BW ratio, upregulated the expressions of SPC and Occludin, alleviated inflammatory infiltration, and repressed inflammatory factor release in the lung tissues. Previous study showed that miR-155-5p-mediated inhibition of differentiation of BMSCs into TypeII pneumocytes promoted the progression of acute respiratory distress syndrome (22). Raut and Khanna (13) reported that miR-23a-3p contributed to the promotion of hepatic transdifferentiation of MSCs. Therefore, our study inferred that upregulated miR-23a-3p in BMSCs might promoted the migration of BMSCs and their differentiation into TypeII pneumocytes to alleviate histopathological abnormalities and inflammatory responses, thus facilitating ALI repair.

MiRNAs can inhibit target mRNA translation or promote target mRNA degradation to modulate various biological functions (34). Our study showed that miR-23a-3p exerted an anti-inflammatory effect in ALI mice. Since FGF2 is a mediator of inflammatory responses (14), we selected FGF2 as a potential downstream mechanism of miR-23a-3p-mediated anti-inflammatory reaction. In our study, bioinformatic and experimental analyses confirmed that miR-23a-3p could directly target FGF2, and we found that BMSCs implantation-induced upregulation of miR23a-3p decreased FGF2 expression in ALI mice, which implied that FGF2 might be involved in miR-23a-3pmediated BMSCs-based lung tissue repair. FGF2 is widely implicated in various biological processes such as cell dif- 
ferentiation, proliferation, and angiogenesis (14). Besides, FGF2 participates in the regulation of autoimmune inflammation (35). Cheng's study discovered that FGF2 overexpression significantly enhanced the release of IL-6 and IL-8 in LPS-stimulated alveolar epithelial cells by inducing the activation of the pro-inflammatory NF- $\kappa \mathrm{B}$ pathway (16). Our study demonstrated that implantation of FGF2-overexpressing BMSCs downregulated the expressions of SPC and Occludin, while increasing the LWW/BW ratio and promoting the release of IL- $1 \beta$, IL-6, IL-4 and IL-8, and it also suppressed the protective effect of miR-23a-3p on the lung tissues. However, the surrounding pericapillary repairs such as pericye activation, as progenitors of MSCs, angintensin II levels, or angiotensin converting enzymes 1-2 was need to further analyzed.

In conclusion, this study revealed that upregulation of miR-23a-3p in BMSCs used for implantation therapy alleviated lung edema and inflammatory infiltration and enhanced lung epithelial permeability through increasing the levels of SPC and Occludin and decreasing the level of inflammatory factors, and thereby promoted the repair of ALI lung tissues. Moreover, inhibiting FGF2 expression in BMSCs used for implantation therapy is the underlying mechanism of miR-23a-3p-mediated BMSCs implantation. However, whether miR-23a-3p directly promoted the differentiation of BMSCs into TypeII pneumocytes should be further validated.

\section{Acknowledgments}

Not applicable.

\section{Potential Conflict of Interest}

The authors have no conflicting financial interest.

\section{References}

1. Confalonieri M, Salton F, Fabiano F. Acute respiratory distress syndrome. Eur Respir Rev 2017;26:160116

2. Bellani G, Laffey JG, Pham T, Fan E, Brochard L, Esteban A, Gattinoni L, van Haren F, Larsson A, McAuley DF, Ranieri M, Rubenfeld G, Thompson BT, Wrigge H, Slutsky AS, Pesenti A. Epidemiology, patterns of care, and mortality for patients with acute respiratory distress syndrome in intensive care units in 50 countries. JAMA 2016;315:788800

3. Cardenes N, Aranda-Valderrama P, Carney JP, Sellares Torres J, Alvarez D, Kocyildirim E, Wolfram Smith JA, Ting AE, Lagazzi L, Yu Z, Mason S, Santos E, Lopresti BJ, Rojas M. Cell therapy for ARDS: efficacy of endobronchial versus intravenous administration and biodistribution of MAPCs in a large animal model. BMJ Open Respir Res 2019;6:e000308

4. Lu H, Cook T, Poirier C, Merfeld-Clauss S, Petrache I, March KL, Bogatcheva NV. Pulmonary retention of adipose stromal cells following intravenous delivery is markedly altered in the presence of ARDS. Cell Transplant 2016;25: 1635-1643

5. Parekkadan B, Milwid JM. Mesenchymal stem cells as therapeutics. Annu Rev Biomed Eng 2010;12:87-117

6. Park J, Kim S, Lim H, Liu A, Hu S, Lee J, Zhuo H, Hao Q, Matthay MA, Lee JW. Therapeutic effects of human mesenchymal stem cell microvesicles in an ex vivo perfused human lung injured with severe $E$. coli pneumonia. Thorax 2019;74:43-50

7. Matthay MA, Calfee CS, Zhuo H, Thompson BT, Wilson JG, Levitt JE, Rogers AJ, Gotts JE, Wiener-Kronish JP, Bajwa EK, Donahoe MP, McVerry BJ, Ortiz LA, Exline M, Christman JW, Abbott J, Delucchi KL, Caballero L, McMillan M, McKenna DH, Liu KD. Treatment with allogeneic mesenchymal stromal cells for moderate to severe acute respiratory distress syndrome (START study): a randomised phase 2a safety trial. Lancet Respir Med 2019;7: 154-162

8. Bartel DP. MicroRNAs: genomics, biogenesis, mechanism, and function. Cell 2004;116:281-297

9. Gu W, Hong X, Le Bras A, Nowak WN, Issa Bhaloo S, Deng J, Xie Y, Hu Y, Ruan XZ, Xu Q. Smooth muscle cells differentiated from mesenchymal stem cells are regulated by microRNAs and suitable for vascular tissue grafts. J Biol Chem 2018;293:8089-8102

10. Li Y, Ke J, Peng C, Wu F, Song Y. microRNA-300/NAMPT regulates inflammatory responses through activation of $\mathrm{AMPK} / \mathrm{mTOR}$ signaling pathway in neonatal sepsis. Biomed Pharmacother 2018;108:271-279

11. Cai ZG, Zhang SM, Zhang Y, Zhou YY, Wu HB, Xu XP. MicroRNAs are dynamically regulated and play an important role in LPS-induced lung injury. Can J Physiol Pharmacol 2012;90:37-43

12. Parker MI, Palladino MA. MicroRNAs downregulated following immune activation of rat testis. Am J Reprod Immunol 2017;77:e12673

13. Raut A, Khanna A. Enhanced expression of hepatocytespecific microRNAs in valproic acid mediated hepatic transdifferentiation of human umbilical cord derived mesenchymal stem cells. Exp Cell Res 2016;343:237-247

14. Beenken A, Mohammadi M. The FGF family: biology, pathophysiology and therapy. Nat Rev Drug Discov 2009;8: 235-253

15. Wullaert A, Bonnet MC, Pasparakis M. NF- $\kappa$ B in the regulation of epithelial homeostasis and inflammation. Cell Res 2011;21:146-158

16. Cheng D, Zhu C, Liang Y, Xing Y, Shi C. MiR-424 overexpression protects alveolar epithelial cells from LPS-induced apoptosis and inflammation by targeting $\mathrm{FGF} 2$ via the NF- $\kappa$ B pathway. Life Sci 2020;242:117213

17. Livak KJ, Schmittgen TD. Analysis of relative gene ex- 
pression data using real-time quantitative PCR and the 2(-Delta Delta C(T)) Method. Methods 2001;25:402-408

18. Li X, Jamal M, Guo P, Jin Z, Zheng F, Song X, Zhan J, Wu H. Irisin alleviates pulmonary epithelial barrier dysfunction in sepsis-induced acute lung injury via activation of AMPK/SIRT1 pathways. Biomed Pharmacother 2019;118: 109363

19. Zhang L, Li Q, Liu W, Liu Z, Shen H, Zhao M. Mesenchymal stem cells alleviate acute lung injury and inflammatory responses induced by paraquat poisoning. Med Sci Monit 2019;25:2623-2632

20. Li L, Dong L, Wang Y, Zhang X, Yan J. Lats1/2-mediated alteration of hippo signaling pathway regulates the fate of bone marrow-derived mesenchymal stem cells. Biomed Res Int 2018;2018:4387932

21. Qin H, Zhao A. Mesenchymal stem cell therapy for acute respiratory distress syndrome: from basic to clinics. Protein Cell 2020;11:707-722

22. Jiang J, Song Z, Zhang L. miR-155-5p promotes progression of acute respiratory distress syndrome by inhibiting differentiation of bone marrow mesenchymal stem cells to alveolar type II epithelial cells. Med Sci Monit 2018;24: 4330-4338

23. Rajasekaran S, Pattarayan D, Rajaguru P, Sudhakar Gandhi PS, Thimmulappa RK. MicroRNA regulation of acute lung injury and acute respiratory distress syndrome. J Cell Physiol 2016;231:2097-2106

24. Cardinal-Fernández P, Ferruelo A, Esteban A, Lorente JA. Characteristics of microRNAs and their potential relevance for the diagnosis and therapy of the acute respiratory distress syndrome: from bench to bedside. Transl Res 2016; 169:102-111

25. Suo T, Chen GZ, Huang Y, Zhao KC, Wang T, Hu K. miRNA-1246 suppresses acute lung injury-induced inflammation and apoptosis via the NF- $\kappa \mathrm{B}$ and $\mathrm{Wnt} / \beta$-catenin signal pathways. Biomed Pharmacother 2018;108:783791

26. Zhang W, Lu F, Xie Y, Lin Y, Zhao T, Tao S, Lai Z, Wei
N, Yang R, Shao Y, He J. miR-23b negatively regulates sepsis-induced inflammatory responses by targeting ADAM10 in human THP-1 monocytes. Mediators Inflamm 2019;2019: 5306541

27. Lecchi C, Dalla Costa E, Lebelt D, Ferrante V, Canali E, Ceciliani F, Stucke D, Minero M. Circulating miR-23b-3p, miR-145-5p and miR-200b-3p are potential biomarkers to monitor acute pain associated with laminitis in horses. Animal 2018;12:366-375

28. Dong L, Li L. Lats2-underexpressing bone marrow-derived mesenchymal stem cells ameliorate LPS-induced acute lung injury in mice. Mediators Inflamm 2019;2019:4851431

29. Wang L, Shi M, Tong L, Wang J, Ji S, Bi J, Chen C, Jiang J, Bai C, Zhou J, Song Y. Lung-resident mesenchymal stem cells promote repair of LPS-induced acute lung injury via regulating the balance of regulatory $\mathrm{T}$ cells and Th17 cells. Inflammation 2019;42:199-210

30. Jin H, Ciechanowicz AK, Kaplan AR, Wang L, Zhang PX, Lu YC, Tobin RE, Tobin BA, Cohn L, Zeiss CJ, Lee PJ, Bruscia EM, Krause DS. Surfactant protein C dampens inflammation by decreasing JAK/STAT activation during lung repair. Am J Physiol Lung Cell Mol Physiol 2018; 314:L882-L892

31. Rao R. Occludin phosphorylation in regulation of epithelial tight junctions. Ann N Y Acad Sci 2009;1165:62-68

32. Zhang YL, Li QQ, Guo W, Huang Y, Yang J. Effects of chronic ethanol ingestion on tight junction proteins and barrier function of alveolar epithelium in the rat. Shock 2007;28:245-252

33. Crosby LM, Waters CM. Epithelial repair mechanisms in the lung. Am J Physiol Lung Cell Mol Physiol 2010;298: L715-L731

34. Afonso-Grunz F, Müller S. Principles of miRNA-mRNA interactions: beyond sequence complementarity. Cell Mol Life Sci 2015;72:3127-3141

35. Shao X, Chen S, Yang D, Cao M, Yao Y, Wu Z, Li N, Shen N, Li X, Song X, Qian Y. FGF2 cooperates with IL-17 to promote autoimmune inflammation. Sci Rep 2017;7:7024 